\title{
Student Drop Tower Competitions: Dropping In a Microgravity Environment (DIME) and What If No Gravity? (WING)
}

\author{
Nancy R. Hall ${ }^{1}$ and Dennis P. Stocker. ${ }^{2}$ \\ NASA Glenn Research Center, Cleveland, Ohio 44135 \\ and \\ Richard DeLombard ${ }^{3}$ \\ Mr. Microgravity ltd, Huron, Ohio, 44839
}

\begin{abstract}
This paper describes two student competition programs that allow student teams to conceive a science or engineering experiment for a microgravity environment. Selected teams design and build their experimental hardware, conduct baseline tests, and ship their experiment to NASA where it is operated in the 2.2 Second Drop Tower. The hardware and acquired data is provided to the teams after the tests are conducted so that the teams can prepare their final reports about their findings.
\end{abstract}

\section{Nomenclature}

$g=$ acceleration of gravity at the Earth's surface $\left(9.81 \mathrm{~m} / \mathrm{s}^{2}\right)$

\section{Introduction}

NASA Glenn Research Center in Cleveland, Ohio conducts two student competition programs, one for highschool-aged students and one for middle school students. The Dropping In a Microgravity Environment (DIME) was held annually for high school teams for six years from school years 2000-2001 through 2005-2006. DIME was restarted for the 2009-2010 school year, continues in the present school year, and is an ongoing program. The inaugural year for the What If No Gravity? (WING) for middle school teams was the 2009-2010 school year. WING is now in its second year and, like DIME, is an ongoing program.

DIME and WING both provide student teams the opportunity to experience a total experimental process from concept development through development, design, construction, operations, data analysis, and writing a final report. This process mimics those processes used by NASA researchers. An adult advisor (most often an educator) guides the student teams throughout the program. ${ }^{1,2}$

Eligibility is open to student teams in all fifty states of the U.S., Washington D.C., Puerto Rico, American Samoa, Guam, Commonwealth of the Northern Mariana Islands, and the U. S. Virgin Islands. These programs extend for most of the school year with student team proposals due at NASA on November 1 each year. The selected teams are announced in early December and those teams then have several months to design, build, and test their experiments before sending them to NASA in early March. The experiments are dropped through the month of March and final reports are due at NASA by late April. ${ }^{3}$

\footnotetext{
${ }^{1}$ Project Manager / Project Scientist, ISS Research Project Office, 21000 Brookpark Road, MS 77-7, and Associate Fellow.

${ }^{2}$ Project Scientist, Combustion and Reacting Systems Branch, 21000 Brookpark Road, MS 77-5.

${ }^{3}$ Consulting Engineer, PO Box 230.
} 


\section{NASA Drop Tower Student Competition}

\section{A. Drop Towers \& Microgravity}

The microgravity environment may be interpreted in many different ways, some proper, some confusing, and some incorrect. Microgravity is not due to zero gravity but rather is due to free fall within a gravitational field. As a result of the various ways to describe the microgravity environment, this has led to misunderstanding and confusion as to what is microgravity. Both DIME and WING allow student to explore this concept and hopefully gain a better understanding of microgravity and its effect on various physical phenomena. ${ }^{4}$

There are several ways to create a microgravity environment that include the International Space Station, the Space Shuttle, sounding rockets, parabolic flight aircrafts and drop facilities. All of the aforementioned methods of creating microgravity conditions rely on free fall. Free fall reduces the effects of gravity so that it appears that gravity has been reduced to zero. For long term microgravity duration lasting several months, experiments are conducted on the International Space Station (ISS). Prior to conducting research on the ISS, experiments were conducted for up to two weeks at a time during Space Shuttle missions. Sounding rockets provide microgravity duration up to 5 minutes, parabolic-flight aircraft provide microgravity research up to 25 seconds and drop facilities provide microgravity research up to 5 seconds. The drop facilities are the most apparent utilizing free fall. NASA Glenn Research Center has two drop facilities for microgravity research: the 2.2 Second Drop Tower and the Zero Gravity Research Facility.

The DIME and WING program utilizes the NASA Glenn 2.2 Second Drop Tower, Figure 1. The tower has been used for nearly 50 years by researchers to study the effects of microgravity on physical phenomena such as combustion and fluid dynamics, and to develop new technology for future space missions. The drop tower's 2.2 second microgravity test time is created by allowing the experiment package to free fall a distance of 79 feet $(24 \mathrm{~m})$. Experiments are integrated into a rectangular aluminum frame and are then enclosed in an aerodynamically designed drag shield on the fifth floor of the tower. This package is hoisted to the top of the tower where the experiment is monitored and controlled before being dropped. The experimental hardware is isolated from aerodynamic drag because it falls within the drag shield. The Education Rig itself falls seven and one half inches $(19 \mathrm{~cm})$ within the drag shield while the entire package is falling. The drop ends when the drag shield and experiment are stopped by an airbag, located at the bottom of the tower. The drop tower is capable of a 45 minute turn-around for experiments that allows for numerous drops during a day.

The two Education Rigs were used for many years to drop demonstration science "experiments" for visitors and are now utilized for the DIME and WING experiments. Each Education Rig, see Figure 2 , provides the following equipment and capabilities: a video camera (30 images per second) with a zoom lens, an electronic data logger with analog and digital inputs, time-delay relays to turn experiment devices on and off at desired times to control an experiment, lighting at the front or back of the experiment, and electrical power at 28 volts and 12 volts for an experiment. The DIME

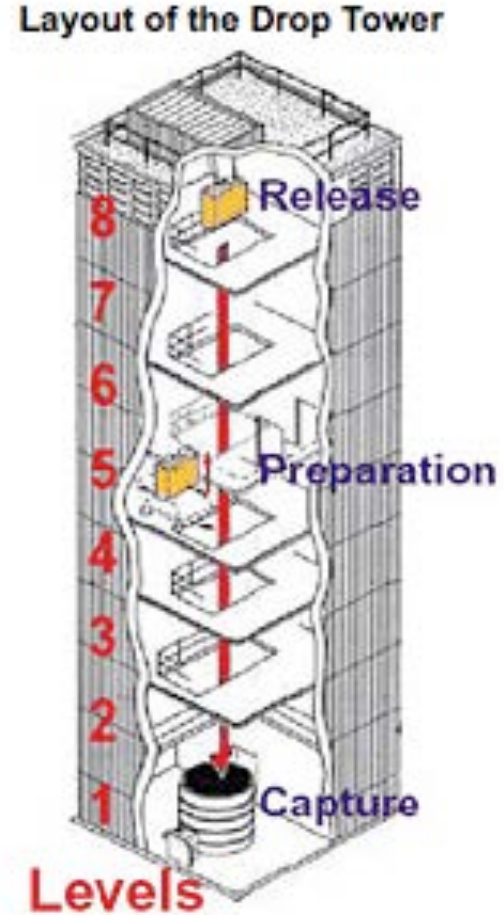

Figure 1. Layout of the NASA Glenn 2.2 Second Drop Tower.

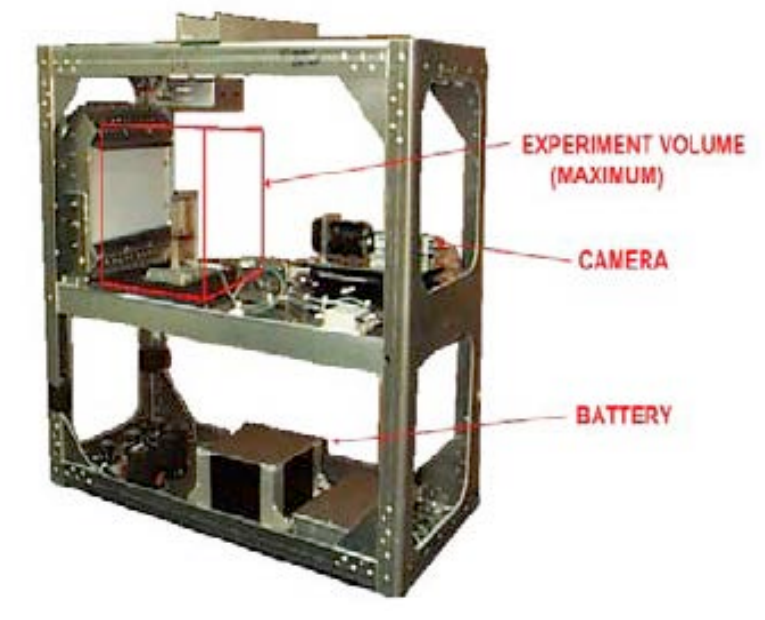

2

Figure 2. Education Rig

American Institute of Aeronautics and Astronautics 
Experiment Design Requirements document ${ }^{5}$ provides important information that can guide the teams in designing experiments.

\section{B. Competition Fundamentals - DIME and WING}

DIME and WING challenge each student team to develop a hypothesis and then design an experiment to test that hypothesis. The DIME and WING program announcement encourages student teams to conduct normal gravity research on their experiment but not all teams are able to do this. The student teams' proposals are submitted to NASA for evaluation by NASA scientists and engineers. The DIME program has two tiers of selected proposals. ,The Tier I teams earn a trip to NASA Glenn to operate their experiments with the NASA staff, while the Tier II team advisors earn a trip to NASA Glenn to help the NASA staff operate their team's experiment. The WING teams will send their experiment to NASA for the NASA staff to operate in the drop tower.

\section{Selection Process}

As proposals are received, they are recorded and given a tracking code that is used during the prescreening and evaluation process. This code allows for evaluation of the proposals without knowledge of the teams' makeup. First, the DIME staff performs a prescreening of the proposals for microgravity effect, safety and meeting the overall rules. The proposals are then evaluated in a blind fashion by a panel of scientists and engineers, i.e., where the evaluators don't know the identity of the proposers.. Multiple evaluators review each proposal, their scores are averaged, and the proposals are sorted to rank the proposals.

Afterwards, each selected DIME team has a mentor assigned from the staff at NASA Glenn to advise and assist a team during the design of the experiment through to the analysis of the results. For WING teams, member(s) of the DIME staff serve as mentor(s) to the WING teams. Each student team is expected to accomplish the work while the mentor lends their expertise in experiment design and drop tower operations to help assure success. It is expected that the mentor will provide "leading questions" to the team as opposed to simply giving answers. For DIME teams, the mentor works with the team representative that visits NASA Glenn and assists teams with interpretation of the acquired data. For WING teams, the data is sent to the school, but teleconferences, etc. are used to discuss the results with the teams.

For non-selected teams, the efforts put forth in microgravity study and scientific research to create a proposal may also be used as a basis for science fair projects. With the team selection announcement in early- December, there is sufficient time for students to expand their work into projects for spring science fairs. These teams may also use the opportunity to critically examine their proposal, their hypothesis, and the experiment concept. The DIME and WING staff does provide comments from the evaluators and are available to provide more detail to the team. We also encourage the student teams to propose the following year. Therefore, the remaining school year time may be spent honing the proposal entry for the following year.

If the sponsoring organization (e.g. high school or science center) has a small drop tower, the team could design and build a smaller demonstration device for that drop tower. If a small drop tower is not available, perhaps the team can channel their interest into building one.

\section{DIME Drop Days and WING Drop Weeks}

DIME Drop Days were initially three days where the four selected teams visited NASA Glenn to drop their experiment, tour center facilities, and attend workshops. However, during the 2005-2006 school year, due to a lack of funding, the student teams paid their own travel costs to visit NASA Glenn. To maximize their time and utilization of the drop tower, each team came for 2 days dedicated to drop tower operations. DIME Drop Days was then split into two two-day segments with two teams working in each segment. The teams enjoyed the one-to-one interaction between the DIME staff and the team as well as increased time working with their experiment. One team member that had participated in DIME in a previous year stated that the present team was more productive during

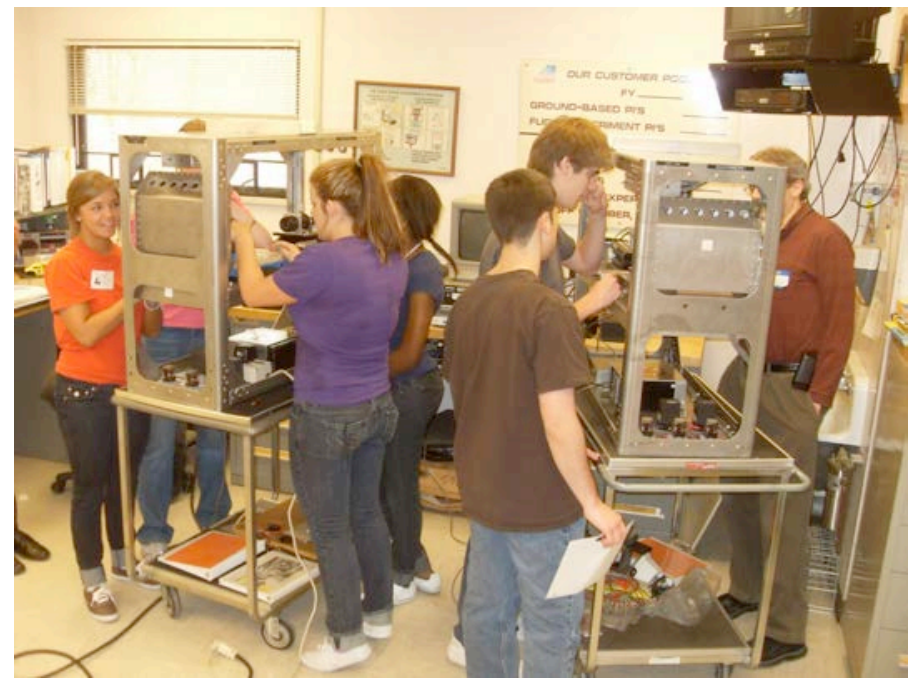

Figure 3. Two teams of students work on their respective experiments mounted in the two Education Rigs. 
those 2 days than for the previous year with 3 days. As a result, when DIME was funded for the 2009-2010 school year, DIME Drop Days was again set up to last 2 days for each team with the day before and/or after available for travel.

Since the WING teams do not come to NASA Glenn to participate in the drops, the DIME staff have some leeway when the drops occur. However, to optimize the technician time and the cost of utilizing the 2.2 Second Drop Tower, several WING experiments are dropped each day of operations over a two week period. The WING team's data are promptly sent to the team along with an initial analysis of the experiment results and the video data.

\section{E. Education standards}

The DIME and WING programs align with the Academic Science Contents Standards in three areas. The first content standard is found in the area of Physical Science. The student investigations allow the students to investigate the concepts of forces and motions that include Newton's laws of motion and gravitational forces. The DIME and WING student experiments are excellent examples of the scientific inquiry where the students can follow the scientific method to design and construct their investigations. Like the scientists at NASA, students must propose their investigation with a design that will gather and analyze data to produce a conclusion. Finally, the DIME and WING program aligns with the Science and Technology standard where students can employ a variety of technologies to gather and present data that describe their observations on the material that are dropped in NASA's Drop Tower.

\section{Dropping In a Microgravity Environment (DIME)}

\section{A. Team Approach}

Experiment design, development, and operation is a team effort at NASA; therefore, this competition asks student teams to detail in their DIME proposal a description of their teamwork in the design, build, development and operation effort of their experiment. The DIME program announcement lists several areas where students can perform specific tasks such as the planning and coordinating of the work, designing the experiment, building the experimental apparatus, conducting experiments as well as communicating the plans and results of the project. This information is to be included in the teams' proposal as well as their plan for accomplishing the work necessary to carry out the proposed experiment, the researching of the topic and writing of the final report. They are asked to describe the variety of skills individual members bring to the team and explain how their team will share an appropriate distribution of workload and

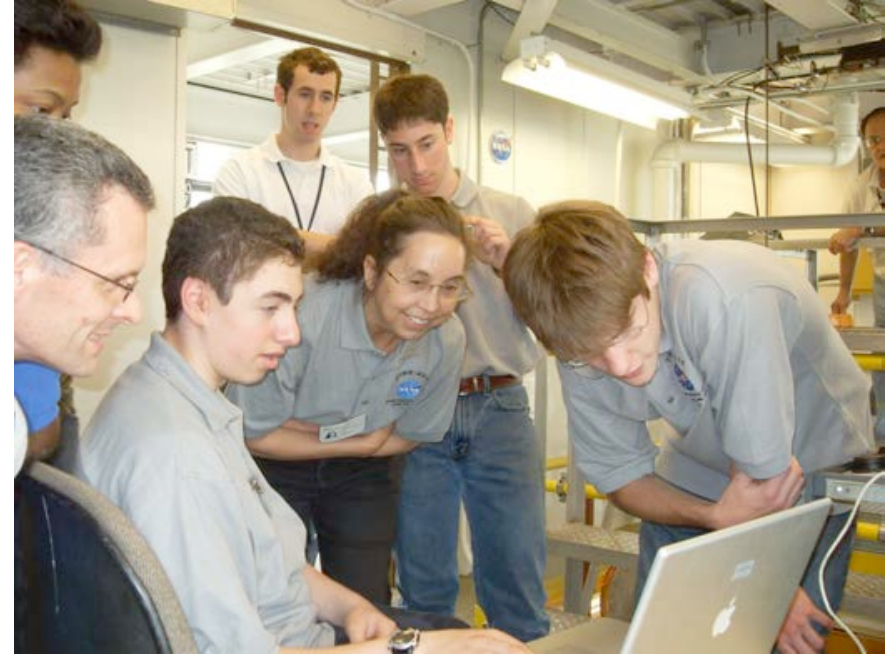

Figure 4. A DIME team reacts while reviewing the video data moments after their experiment was dropped in the $\mathbf{2 . 2}$ Second Drop Tower. responsibilities. In the drop tower during DIME Drop Days, it has been observed that successful teams have members with assigned responsibilities.

All these different job functions show that it's not only the math and science students that can be involved in a DIME team. Technology students can help with the preparation of the conceptual drawings in the proposal as well as the design drawings once the team is selected. English or computer students with the skills in page layout and word processing are necessary due to the specific rules for preparing a proposal, including page layout format, number of pages, and technical content. Science students would conduct the baseline $1 \mathrm{~g}$ testing and math students could perform the analysis. Alternatively, any student with an interest could participate in any area in which they may contribute.

Both the mentor and adult advisor also have roles in the team. Both should guide the students, but not perform the work for the students. 


\section{B. Design and Fabrication of Experiment}

Following their selection, a NASA mentor is assigned to guide the team as the team works through the more difficult stage of design, fabrication, and testing.

NASA furnishes each selected DIME team a base plate and electrical connectors (if required) that together form the interface with the NASA rig used in the drop tower. The teams begin to develop the design of their experiment in more detail and submit a preliminary design to NASA for review. This review is to assess the technical aspects of the experiment design for proper interface with the NASA rig, adherence to safety requirements, expected acceptable operation in the drop tower, and to make sure each team is progressing (and not waiting for the last minute).

One month later, the teams submit their final design primarily for review of compliance with the safety requirements. The NASA mentor provides feedback to the team after a design review by the NASA DIME staff. When construction is completed, the experiment is shipped to NASA Glenn. A safety team inspects the experiment apparatus and its design package before it will be operated in the drop tower. Safety is a high priority in the drop tower and student experiments are naturally expected to meet the applicable safety standards, as are all other experiments.

\section{Student Experiments}

The DIME experiments over the years have ranged from simple to complex, failures to successes, physical demonstrations of forces and motions to fluid flow to combustion, and many variations between these extremes. While many students' experiments over the years have been successful, there have also been some failures. This is wholly not unexpected in these student experiments just as it is typically experienced in both the NASA and academic experiments that have been brought into the 2.2 Second Drop Tower. Many teams have often learned much more by experiencing initial failure of their experiment to operate as they expected. The process of diagnosis, analysis, and corrective action is very educational, especially when it is repeated several times before success is achieved! It must be stated that the NASA staff (scientists, engineers and technicians) do actively assist the teams in their corrective actions, as required, but such work is still directed by the students.

The essence of a few experiments is described here. Some DIME experiments have been simple demonstrations of mechanical motion, such as small ball bearings rolling around in a funnel when the experiment is dropped. The motion is at first unexpected, but is easily followed after simple analysis. Some simple experiments in capillary flow have been popular to measure the rate of rise of oils in capillary tubes. A surprising simple experiment was able to create free-floating droplets of water from simple capillary action. More complex experiments have included pressurized small plastic bottles to enable water flow from nozzles. A complicated experiment consisted of two separate chambers containing granular sand that fell in an electric field during the microgravity period. The experiment included electrical charge circuitry as well as solenoid controls. A multi-faceted fluid experiment has a droplet generator and a large fluid blob system. A control system started droplets falling before the experiment was released and a system also popped a water balloon during the microgravity time. A simple apparatus was made in a double chamber box with two different dish detergents inside. Two copper loops were rotated before the drop to get a thin soap film in the loop. Destruction interference was observed with white light before and during the drop that indicated the film thickness in 1-g and in microgravity.

\section{DIME Success Stories}

There are many instances of past DIME students continuing on into engineering and science programs in college. Feedback from students and teachers has always been positive at the end of DIME, even years later when they are in college. Examples of feedback received after DIME Drop Days include the following from student, "Learning how to think critically and not being disappointed with unexpected results. Another very beneficial part was talking to all of the NASA workers and learning that it's not impossible to become an engineer."

A teacher commented, "My students talked all the way home about the feelings they had when their experiment WORKED for their second and third drops! It was priceless! They said that the eight months of work that they put into their experiment were absolutely worth it. We may have four more young people on their way to careers in research science because of the DIME experience!"

Another teacher commented "The DIME experience doesn't end with the four girls who were able to attend DIME Days from our school. Their enthusiasm and excitement have carried over to the rest of the school- they have told everybody about their experiment and their week at NASA Glenn! I already have students talking about what they're going to propose next year!"

A student relayed to the DIME staff that he was changing his college major to engineering after his experience with DIME. 
Another student was a member of two winning teams in successive years. Since DIME rules allow only one repeat team member, she gathered other students the next year to form another team. During her second year at DIME Drop Days, she expressed an interest in becoming an astronaut. She later entered college at Purdue University and obtained a co-op position at NASA Johnson Space Center. The co-op work helped focus her studies in propulsion. She is currently working for Boeing in the area of wind turbines. In February 2010 she had this to say about DIME, "I am so excited that DIME has been started up again (and expanded!). It was such a great experience in high school. Hopefully there will be many other students who benefit from it as I did."

One high school educator incorporated the process of creating DIME proposals into her AP Physics class at the beginning of each school year. She pre-selected four proposals that were then submitted to NASA as DIME entries. She had three teams succeed in three successive years, which, by some coincidence were comprised of four (different) young ladies each year.

One DIME team was frustrated by their experiment and their lack of success to achieve suspension by acoustic levitation. They tried many remedies during their two days in the drop tower but were never able to claim victory. One team member was so challenged that he pursued acoustic levitation in college in the following year. He got friends to help him assemble a much larger acoustic chamber and with more research found that the DIME team had tried to levitate at the wrong position in the acoustic wave! He was then successful with the college acoustic chamber. When he returned to NASA Glenn the following summer as an undergraduate intern, he proudly showed the NASA DIME staff a video of the successful operation.

\section{What If No Gravity? (WING)}

\section{A. Team Approach}

As with DIME, the WING program encourages team work so that each student team includes sufficient students with the varied skills involved in planning of the work to be done, definition of scientific principles, experiment design, building and testing, as well as communicating the results of the project. While middle school students may not have, for example technology or computer skills, there are still students who excel in certain areas and can contribute in these specific areas.

In addition, the role of the teacher/adult advisor is more collaborative with the student teams than for a high school student team. These students may need additional help, guidance and/or supervision with the construction of the experiment apparatus. In addition, proofreading of the proposal, discussions on microgravity as well as detailed discussion of the scientific process in grades 5-8 will provide the student with the necessary background to allow them to continue on through high school into the DIME program and readily take the lead in doing the research on up through the analysis. The NASA mentor role here is similar to that with DIME. They help guide and support the teacher/student team.

\section{B. Design and Fabrication of Experiment Hardware}

Following their selection, a NASA mentor is assigned to guide the team as the team work through the more difficult stage of design, fabrication, testing and data analysis.

NASA provides and generally requires use of a clear plastic box within the Education Rig to contain the WING experiments. This relieves the teams from the need to build such an enclosure as a secondary fluid containment vessel. A set of relay contacts is also available for simple electrical control of their experiment during drop operations, e.g., to activate an electric device during free fall.

The teams begin to develop the design of their experiment in more detail and submit a preliminary design to NASA for review. This review is to assess the technical aspects of the experiment design for proper interface with the NASA rig, adherence to safety requirements, expected acceptable operation in the drop tower, and to make sure each team is progressing (and not waiting for the last minute).

Later, the teams submit their final design primarily for review of compliance with the safety requirements. When construction is completed, the experiment is shipped to NASA Glenn. The WING staff inspects the experiment apparatus and its design package before it will be operated in the drop tower. Safety is a high priority in the drop tower and student experiments are naturally expected to meet the applicable safety standards, as are all other experiments.

\section{Student Experiment Summary}

The experiments in WING 2010 had a wide variation of complexity ranging from a suspended Slinky TM to a bottle of water with a ping pong ball inside to a large mass moved by stretched springs to double electromagnets with iron filings, Figure 5. 
There were several WING 2010 experiments that dealt with the capillary rise of a fluid to contact two wires to complete a circuit to light a lamp. This simple sort of experiment involved fluid study as well as electrical design and construction.

Probably the most surprising WING 2010 experiment was one of the most simple being comprised of a bottle of water with a colored ping pong ball inside. Normally, the expectation is that the ball remains floating on the top surface of the water during microgravity. The ball was drawn down into the water the moment the experiment was dropped! To increase the visibility of the ball in their tests at school, the students had colored the ping pong ball with an ink marker. The students had unknowingly chosen an ink with wetting characteristics (i.e., shallow contact angle) that made the water envelope the ball, effectively pulling the ball "under water."

\section{WING Success Stories}

Being only in its second year, the WING success stories aren't as numerous as the DIME success stories and neither has this program been around long enough to see how it has shifted student's planning for educational goals but some impact has already been made.

In the WING inaugural year, a curriculum director conducted a workshop with over 100 middle-school educators and students in the fall of 2009 to (among other topics) familiarize the participants with the WING competition. Two NASA WING staff members participated in an hour-long videoconference with the participants during the workshop to explain microgravity, describe the WING competition, and answer questions. Fourteen of the eighteen WING 2010 projects came from that workshop. Several of those educators have followed up with WING 2011 proposals.

\section{Scientist \& Engineer Contribution}

While the DIME and WING are research programs, they also introduce the students to the multidiscipline approach that research teams take to tackle a problem. Besides the mentoring engineers and scientists, other engineers and researchers are encouraged to provide insight into the design of the experiment after its selection.

Scientists and engineers may help by introducing DIME and WING to their local schools and/or educators. Promoting DIME and WING helps contribute to students learning and experiencing the scientific process and helping to expand STEM in the schools. Scientists and engineers may also serve as professional mentor for local teams. A scientist, engineer, or parent may also form a team outside of a formal school setting and act as the adult advisor for the team. There is no program requirement for a team to be based at a school. The NASA DIME and WING staff expect the student teams to do the real work, but professional advice is quite often helpful if a team is stuck.

\section{Conclusion}

The NASA Strategic plan lists among its goals to promote Science, Technology, Engineering, and Mathematics (STEM) literacy through strategic partnerships with formal and informal organizations and to inform, engage and inspire the public by sharing NASA's missions, challenges, and results. By participating in DIME and WING, students, educators and the public help NASA meet these goals.

\section{Acknowledgments}

The DIME and WING programs are funded by the Teaching From Space Office at the NASA Johnson Space Center, Houston, Texas. The Teaching From Space Office facilitates education opportunities that use the unique environment of human spaceflight. 
The authors thank Dawn Jenkins (QinetiQ NA at NASA Glenn) for assistance and advice and Arela Leidy (Ohio Aerospace Institute) for her assistance with coordinating the travel, logistics and shipment of experiment hardware for the DIME and WING teams.

The authors also thank the NASA and National Center for Space Exploration Research scientists and engineers who have reviewed the student teams' proposals and have served as mentors to the student teams.

The authors are especially grateful to the 2.2 Second Drop Tower technicians who support the student teams during their visit.

The DIME and WING staff also appreciate the support of the 2.2 Second Drop Tower facility mangers, Eric Baumann and Eric Neumann.

Finally the authors want to thank the students, teachers, and other adult advisors who have participated in DIME and WING over the years, for without them, this program would not be possible.

\section{References}

${ }^{1}$ DIME Program Announcement, NASA Glenn Research Center, http://spaceflightsystems.grc.nasa.gov/DIME_Documents/DIME_2011_PA.pdf

${ }^{2}$ WING Program Announcement, NASA Glenn Research Center, http://spaceflightsystems.grc.nasa.gov/DIME_Documents/WING_2011_PA.pdf

${ }^{3}$ DIME web page, http://microgravity.grc.nasa.gov/DIME.html

${ }^{4}$ DIME \& WING Educators Resource Guide http://spaceflightsystems.grc.nasa.gov/DIME_Documents/DIME_2010_ERG.pdf

${ }^{5}$ DIME Experiment Design Requirements http://spaceflightsystems.grc.nasa.gov/DIME_Documents/DIME_2010_EDR.pdf 